\title{
Hemoperitoneum following a Motor Vehicle Accident in a Patient with 20 Uterine Leiomyomas
}

\author{
Gregory $\mathrm{Wu}^{\mathrm{a}}$ Olivier Urayeneza ${ }^{\mathrm{b}} \quad$ Gudata Hinika $^{\mathrm{b}}$ \\ aRoss University School of Medicine, Miramar, FL, USA; ${ }^{b}$ Department of Surgery, Dignity \\ Health California Hospital Medical Center, Los Angeles, CA, USA
}

\section{Keywords}

Leiomyoma · Hemoperitoneum · Trauma surgery · Hysterectomy

\begin{abstract}
Uterine leiomyomas are neoplasms of the smooth muscle that can cause complications such as severe bleeding and infertility in women of reproductive age. While many individuals may be asymptomatic, others may present with anemia secondary to heavy bleeding, cyclical abdominal pain, pelvic pressure, and urinary or bowel symptoms. A rare complication of uterine leiomyomas is avulsion due to blunt abdominal trauma resulting in hemoperitoneum. We present a 49-year-old female with no pertinent medical history who presented to the emergency room following a motor vehicle accident. Computed tomography scan revealed extensive hemoperitoneum and the patient was taken to the operating room where the source of bleeding was identified as multiple avulsed leiomyomas. The patient underwent an emergency hysterectomy and bilateral salpingectomy. Pathology reported a uterus weighing 6,000 $\mathrm{g}$ and the presence of 20 leiomyomas, with the largest measuring $29 \mathrm{~cm}$. Knowledge of leiomyoma symptoms, presentation, and complications by both the patient and clinicians may help identify diagnoses and expedite intervention in the emergency setting.
\end{abstract}




\section{Introduction}

Uterine leiomyomas, also known as fibroids, are benign tumors of the myometrium and are the most common pelvic tumor in females. The prevalence of uterine leiomyoma in women over the age of 45 years is greater than $60 \%$. Fibroid symptoms can be categorized into three groups: (1) heavy/prolonged bleeding, (2) pelvic or abdominal pressure, and (3) reproductive dysfunction [1]. While most patients are diagnosed as a result of their symptoms or by incidental radiological findings, patients with fibroids are often asymptomatic and undiagnosed. Because fibroids are estimated to present symptomatically in only $25 \%$ of women of reproductive age, a large majority of patients with fibroids are actually unaware of their condition [2].

Blunt abdominal trauma (BAT) from a cause such as a motor vehicle accident may result in injury to the small bowel, large bowel, liver, spleen, or vasculature. Intra-abdominal injuries following BAT are reported to be as high as 12-15\% [3]. BAT may cause a shearing force that can lacerate organs, stretch vessels, and create tears at vascular pedicles. These mechanisms of injury may result in hemoperitoneum and/or organ infarction. Hemoperitoneum may present as acute abdominal pain, diffuse tenderness and rigidity upon palpation of the abdomen, and hemodynamic instability. A focused assessment with sonography (FAST) is often performed, which can identify free fluid in the abdomen. Patients with BAT that have signs of peritonitis or are hemodynamically unstable with a positive FAST should undergo emergency laparotomy to identify and control the source of bleeding.

Herein, we report a rare complication of uterine leiomyomas causing hemoperitoneum secondary to BAT and discuss its presentation, pathogenesis, management, and treatment.

\section{Case Report}

A 49-year-old woman with no significant medical or gynecological history presented to the emergency room as a level I trauma following a motor vehicle accident while traveling at 40 miles/h. The patient appeared to be in distress and complained of diffuse abdominal pain. She was hypotensive upon arrival to emergency department but responded to initial resuscitation with fluids and 2 units of pRBC. Physical exam revealed positive seatbelt sign as well as abdominal peritoneal irritation upon palpation. A FAST exam was positive in the right upper quadrant at Morrison's pouch. Hemoglobin was $11.2 \mathrm{~g} / \mathrm{dL}$. The patient was taken for a computed tomography (CT) scan, which revealed free abdominal fluid as well as a large intra-abdominal and pelvic mass measuring $34.4 \times 24 \times 15 \mathrm{~cm}$ (Fig. 1). The patient was immediately taken to the operating room for emergency laparotomy, as she became hypotensive again after initial resuscitation.

A midline incision was made from the xiphoid to the pubis and revealed a large amount of blood. All quadrants were packed and subsequent removal of packings from the lower quadrants revealed multiple space-occupying solid masses. Careful evaluation established that bleeding was from multiple pedunculated fibroids. At this point, an intra-operative obstetric/gynecological consult was obtained. Bleeding was noticed from the lower base of the pedunculated fibroids in the posterior uterus. The decision was made to perform a hysterectomy with bilateral salpingectomy. Hemostasis was achieved, the abdomen was closed, and the patient was transferred to PACU in a stable condition.

The pathology report identified a myomatous uterus weighing 6,030 $\mathrm{g}$ and measuring $42 \times 42 \times 15 \mathrm{~cm}$ (Fig. 2). A total of 20 fibroids were identified macroscopically and 
histologically (11 subserosal, 2 submucosal, 7 intramural) with the largest fibroid measuring $29 \mathrm{~cm}$. The patient had an uneventful postoperative course and was discharged on postoperative day 5 .

\section{Discussion/Conclusion}

Uterine leiomyoma commonly manifests in women of reproductive age as heavy or prolonged menstrual bleeding. However, intra-abdominal bleeding due to uterine leiomyomas is a rare finding, with less than 100 reported cases in the literature. Most of these reported cases of hemoperitoneum are due to either spontaneous or traumatic rupture of leiomyoma. Spontaneous rupture of leiomyomas is caused by disruption of overlying veins or arteries. Rupture of these veins may be due to increased venous pressure or increased intra-abdominal pressure, caused by conditions such as menstruation or pregnancy, or secondary to actions of heavy lifting [4]. Spontaneous arterial rupture may be caused by hypertension or large fibroids that overstretch arterial vessels [5]. On the other hand, traumatic rupture of leiomyoma can occur when an external traumatic force, such as a vehicle collision, induces a contrecoup-like mechanism within the pelvic organs. On impact, the differences of size and speed between the uterus and fibroids cause a shearing force that causes the vessels to overstretch and tear at their pedicles [6]. Obvious organs vulnerable to this mechanism include the liver, spleen, and bowel; however, the avulsion of leiomyoma should also be included in the differential of a woman who presents post-trauma with abdominal or pelvic pain.

The symptoms and management of fibroids may differ depending on the size, location, type, and number present. An increase in size and number can result in bulk symptoms by causing pressure on the adjacent structures such as the bowel, bladder, ureters, and vasculature. A large fibroid may also cause increased stretching of vessels overlying its surface. Additionally, research by Lai et al. [7] suggests that the pedunculated subtype of leiomyoma is at increased risk for many complications, including acute torsion and hemorrhagic infarction from the twisting of the leiomyoma around a thin stalk. All things considered, our patient with 20 leiomyomas (mostly pedunculated), with the largest measuring $29 \mathrm{~cm}$, had significant risk factors for complications. It is no surprise then that shearing forces from BAT could cause her 6,000-g myomatous uterus to avulse and cause extensive hemoperitoneum.

In an asymptomatic patient, the diagnosis of uterine leiomyoma is often missed. When classic symptoms present, diagnosis is safely and cost-effectively made by ultrasound. However, radiological diagnosis of hemoperitoneum due to leiomyoma may pose a challenge. In a patient who presents to the emergency room with an acute abdomen and stable vitals, a FAST exam should be performed, and if positive, followed by a CT scan to identify the source and extent of bleeding to prepare for surgery. However, ultrasound and CT scan have their limitations in identifying leiomyomas as the cause of hemoperitoneum, as fluid can obscure important structures for diagnosis. In our patient, a FAST ultrasound revealed a large pelvic mass with surrounding free fluid. A subsequent CT scan showed a pelvic mass in which malignancy was proposed but was not able to be specified due to overlying free fluid. The presence of fluid plus unstable vitals was an indication to proceed with surgical intervention.

While clinical presentation, radiology, and surgical exploration are necessary for the diagnosis of avulsed leiomyomas, the patient's history is paramount to the overall clinical picture. In our case, the patient had reported no gynecological history or previous surgeries. As the CT scan could not clearly identify the site of bleeding, it was not until exploratory 
laparotomy that multiple large, pedunculated fibroids were found and identified to be the source of bleeding. It was then that an intraoperative consult to gynecology was made for intervention.

The standard management of symptomatic leiomyomas include myomectomy, myolysis, embolization, and drugs such as GnRH agonists [8]. Removal of the fibroids often leads to resolution of symptoms. If symptoms persist, repeat embolization or hysterectomy may be considered (the most common indication for a hysterectomy are uterine fibroids) [1]. In the emergency setting, a patient with acute uterine hemorrhage due to fibroids may undergo a hysterectomy or percutaneous embolization of the internal iliac arteries. Our patient underwent a complete hysterectomy and bilateral salpingectomy as she expressed no further desire for childbearing. Furthermore, there is an increased risk of sarcomatous degeneration of leiomyomas at her age [9].

In summary, clinicians should be aware that hemoperitoneum due to traumatic avulsion of leiomyomas is a rare, but serious, complication. Radiographical studies aid in the diagnosis but may be unable to delineate the origin of bleeding, as seen in our patient [10]. Therefore, diagnosis is challenging and requires the interaction of patient history, clinical presentation, and radiography to consider this differential. We propose that patients should be educated on the typical and atypical presentation of fibroids, as this knowledge could assist clinicians and radiologists to a timely diagnosis, allow earlier gynecological consult, and expedite surgical intervention in the setting of life-threatening hemoperitoneum.

\section{Statement of Ethics}

The authors have no ethical conflicts to disclose. Informed consent was obtained from the patient for the publication of this case report. There are no patient identifiers in the case report which may link the patient to the report.

\section{Disclosure Statement}

The authors have no conflicts of interest to declare.

\section{Funding Sources}

The authors did not receive any financial support.

\section{Author Contributions}

G.W. drafted the manuscript. O.U. performed the emergency general anesthesia surgery. O.U. and G.H. read, reviewed, and approved the final transcript. 
Wu et al.: Uterine Leiomyomas Causing Hemoperitoneum Secondary to BAT

\section{References}

1 Stewart EA. Clinical practice. Uterine fibroids. N Engl J Med. 2015 Apr;372(17):1646-55.

2 Stewart EA, Cookson CL, Gandolfo RA, Schulze-Rath R. Epidemiology of uterine fibroids: a systematic review. BJOG. 2017 Sep;124(10):1501-12.

3 Boutros SM, Nassef MA, Abdel-Ghany AF. Blunt abdominal trauma: the role of focused abdominal sonography in assessment of organ injury and reducing the need for CT. Alexandria J Med. 2016;52(1):3541.

4 Buttery BW. Spontaneous haemoperitoneum complicating uterine fibromyoma. Aust N Z J Obstet Gynaecol. 1972 Aug;12(3):210-3.

5 Swarray-Deen A, Mensah-Brown SA, Coleman J. Rare complication of fibroids in pregnancy: spontaneous fibroid rupture. J Obstet Gynaecol Res. 2017 Sep;43(9):1485-8.

6 Dasari P, Maurya DK. Hemoperitoneum associated with fibroid uterus. J Obstet Gynecol India. 2005;55(6):553-4.

7 Lai YL, Chen YL, Chen CA, Cheng WF. Torsion of pedunculated subserous uterine leiomyoma: A rare complication of a common disease. Taiwan J Obstet Gynecol. 2018 Apr;57(2):300-3.

8 Mizrahi DJ, Kaushik C, Adamo R. Hypovolemic Shock and Hemoperitoneum from Spontaneous Avulsion of a Large Pedunculated Uterine Leiomyoma. J Radiol Case Rep. 2017 Mar;11(3):15-21.

9 Prayson RA, Hart WR. Pathologic considerations of uterine smooth muscle tumors. Obstet Gynecol Clin North Am. 1995 Dec;22(4):637-57.

10 Kamat NV, Telkar HB, Ramani SK, Thakker AP. Ruptured degenerated uterine fibroid diagnosed by imaging. Obstet Gynecol. 2001 Nov;98(5 Pt 2):961-3.
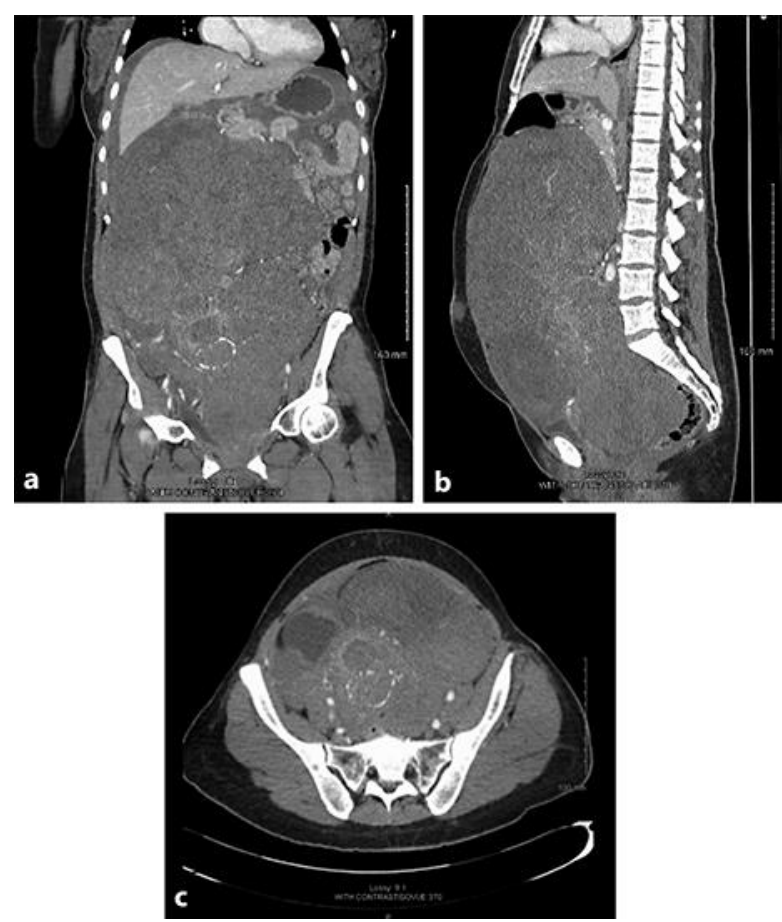

Fig. 1. a-c Abdominal CT scan revealing large intra-abdominal and pelvic mass with associated fluid collections suggesting hemoperitoneum. 

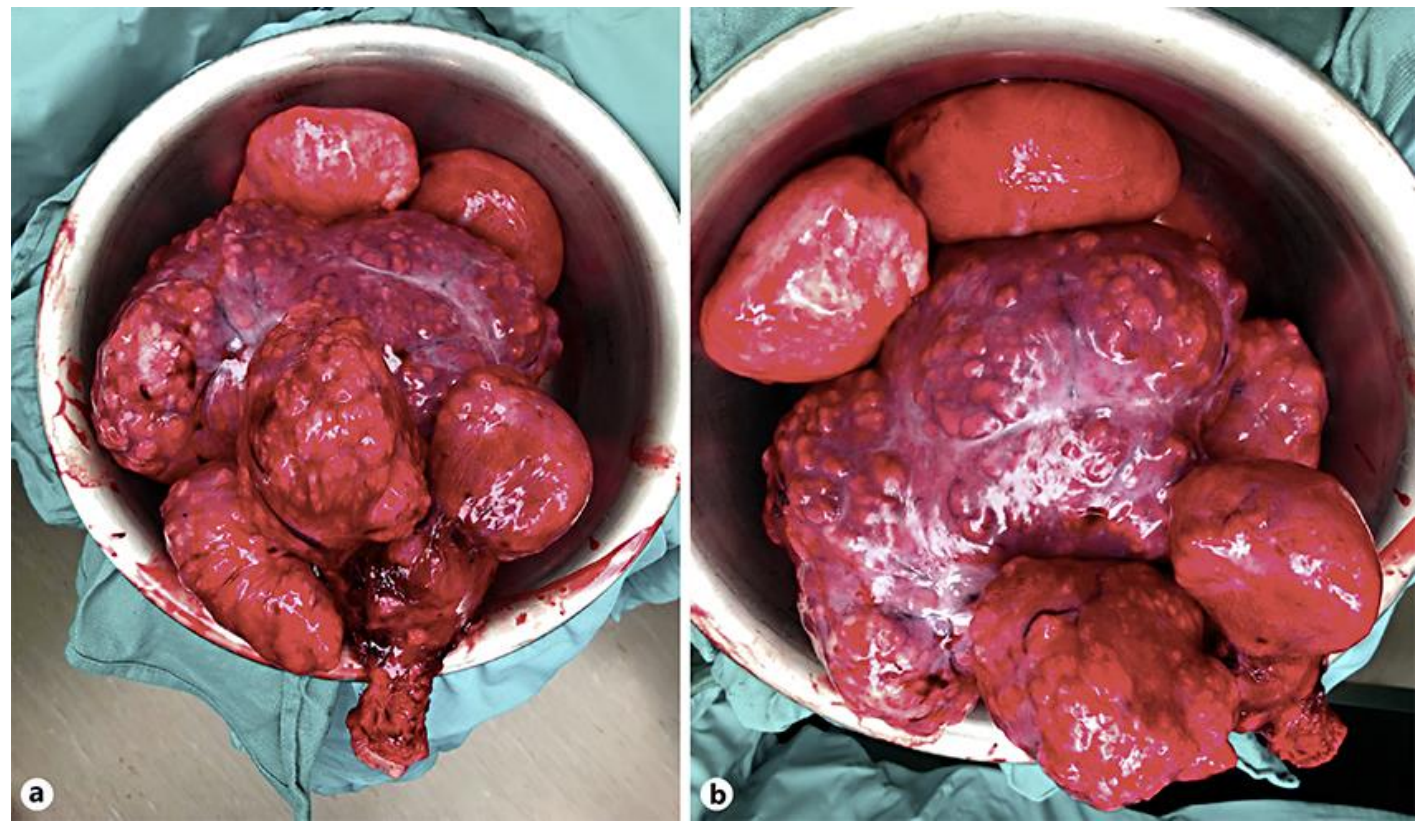

Fig. 2. a, b Intraoperative images of a myomatous uterus with multiple enlarged pedunculated fibroids following an emergency hysterectomy. 